\title{
Spatial Data Performance Test of Mid-cost UAS with Direct Georeferencing
}

\author{
Branko Kordić ${ }^{*}$, Mateo Gašparović², Borna Lužar Oberiter ${ }^{3}$, Almin Đapo ${ }^{4}$, Goran Vlastelica ${ }^{5}$ \\ ${ }^{1}$ Croatian Geological Survey, Sachsova 2, Zagreb 10000 \\ ${ }^{2}$ Chair of Photogrammetry and Remote Sensing, Faculty of Geodesy, University of Zagreb, Zagreb 10000, Croatia \\ ${ }^{3}$ Department of Geology, Faculty of Science, University of Zagreb, Zagreb 10000, Croatia \\ ${ }^{4}$ Chair of Hydrography, Faculty of Geodesy, University of Zagreb, Zagreb 10000, Croatia \\ ${ }^{5}$ Faculty of Civil Engineering, Architecture and Geodesy, University of Split, Matice hrvatske 15, Split, Croatia \\ * Corresponding author, e-mail: bkordic@hgi-cgs.hr
}

Received: 23 January 2020, Accepted: 04 May 2020, Published online: 18 June 2020

\begin{abstract}
Recent development of lightweight and small size multi-frequency GNSS receivers allows determination of the precise position of the moving platform and spatial data acquisition without the need for setting up and measuring of ground control points. The main advantage of this approach is a higher operational capacity with reduced time and cost of field measurement. This relates to fieldwork in inaccessible areas with demanding terrain configuration. In this paper development and use of a UAS with direct georeferencing of camera sensor for spatial data acquisition is described, and the possibility of 3D scene reconstruction based on the precise position of the camera with predetermined interior parameters is examined. Modern computer vision-based SfM photogrammetry algorithms are used for determining attitude parameters and reconstruction of the scene. For that purpose, several tests on two different test fields were performed using various system parameters for collecting and analysis of several spatial data sets. The presented results demonstrate a satisfactory accuracy $(3.1 \mathrm{~cm}$ planar and $6.4 \mathrm{~cm}$ spatial) of the system for various applications in geodesy.
\end{abstract}

Keywords

UAS, geodesy, direct georeferencing, SfM photogrammetry, spatial accuracy, GCP

\section{Introduction}

Unmanned vehicles are a very popular topic that has found its application in various areas of human exploration. Nowadays, there are many different Unmanned Aircraft System (UAS) solutions on the market, which are commercially available and can be used for high-resolution geodetic surveying and mapping [1, 2]. Most of these systems are equipped with Global Navigation Satellite System (GNSS) receivers using stand-alone and differential codephase ambiguities correction solutions. With these receivers, it is possible to determine the initial position of images with a meter or sub-meter level of accuracy [2]. In order to achieve survey-grade accuracy of few centimetres with such systems, and to produce high-resolution spatial data derived information such as the orthomosaic, digital elevation model (DEM) or point cloud it is necessary to stabilize and measure ground control points (GCPs) [3]. Coordinates of GCPs are measured with geodetic survey-grade GNSS with centimetre level accuracy and used to compute the correct position and orientation of the camera sensor. This method is also known as Integrated Sensor Orientation (ISO). GCPs can be made in different shapes and sizes, depending on altitude and Ground Sample Distance (GSD) or required accuracy. Accuracy of GCPs directly affects the accuracy and quality of the processed data and final output [2-5]. A total station (TS) can be used for determination of GCPs with millimetre level accuracy. In any event, setting up of GCPs in certain field conditions and terrain configurations can be costly. The time required for this activity considerably prolongs the time and expense of fieldwork. In most cases installation, measurement and collection of GCPs last several times longer than the flight time and capturing of images with a UAS.

Recently, there has been increased development of MST (Micro System Technology) and growth of commercial UAS solutions, which use miniature and lightweight GNSS devices with carrier-phase ambiguities solution that 
can determine 3D positions at the level of a few centimetres [6]. For Direct Sensor Orientation (DSO) it is necessary to know the position and attitude of the sensor in a certain moment of time (tPA) and the interior camera calibration parameters. Derived direct image orientation parameters contain errors due to inaccurate determination of the relative orientation between reference frames of different sensors [7]. Furthermore, a moving platform inclines at different angles and directions, which leads to differences between the reference frame of the GNSS antenna and the camera sensor. Relative attitudes between reference frames cannot be taken as a constant value. Relative attitudes between GNSS, Inertial Measure Unit (IMU) and the camera can be unstable [8]. The orientation of the camera sensor can be estimated based on IMU data integrated into the flight controller and magnetometer [9], but the accuracy of this low-cost sensor is not sufficient for accurate attitude determination [10]. Geodetic survey grade attitude precision with tactical grade lightweight IMU are not sufficiently represented for now, but we can expect significant progress in the future [1]. Nowadays there are lightweight DSO solutions on the market designed for UAS application, but they are still expensive with questionable cost-benefit [11-13].

In this paper, the capabilities of the cost-effective UAS for application in geodesy is explored. In the presented approach position and interior camera parameters are used as input parameters, which serve as initial values for automatic scene reconstruction. Input position of the camera is corrected based on IMU data of the flight controller. The exterior orientation of images is computed by structure from motion (SfM) multi-view stereo (MVS) algorithms implemented in modern photogrammetric software special designed for processing of aerial images from UAS [14-18]. Field tests were performed at two sites. The first site was at a flight test polygon in Slavonski Brod in Croatia, which served as a calibration site. The second site is located along the coast of Krk Island and contains natural features and structures, which represents a typical survey site.

\section{Methods}

\subsection{Unmanned aircraft platform}

A custom-made VTOL (Vertical TakeOff and Landing) multi-rotor system is used as the aerial platform for all the flights of our test process. The system is made from a carbon fibre tube frame with a radial setup of engines powered with a high capacity Lithium Polymer (LiPo) battery.
The total weight of the system is about $3.6 \mathrm{~kg}$, classifying it as a micro aerial vehicle (MAV) with weight less than $5 \mathrm{~kg}$ (Fig. 1). The flight time of the system in normal conditions is about $26 \mathrm{~min}$. Apart from the GNSS receiver and the camera, the platform is equipped with a Pixhawk flight controller (version: 1.8.2.) that includes additional sensors such as gyroscopes, accelerometers, 3 -axis magnetometer and barometer used for navigation support. The reason for using multi-rotor VTOL is its ability to capture images from different positions, applicability in many specific tasks, the possibility of launching and landing with the minimal required area, as well as flight stability and operation at lower altitudes as compared to light fixed-wing systems without VTOL.

UAS is monitored and controlled by a ground station using dual commands via RF link. An essential component for the aerial survey is open source software Mission planner (version: 1.3.50.0, firmware: APM: Copter 3.4.4) used for planning purposes, control and real-time management of UAS.

\subsection{UAS positioning systems}

Most UAS s use low-cost single-frequency GNSS receiver that can receive signals from GPS, GLONASS and Galileo satellites [19-23]. Such devices allow positioning with an accuracy at the meter level. Based on differential GNSS with Code-phase ambiguity corrections, sub-meter accuracy can be obtained [24]. In order to achieve such centimetre level accuracy, it is necessary to stabilize and measure GCPs in the field. In recent years, single-frequency GNSS receivers have appeared on the market, which allows the determination of the 3D trajectory of moving objects with an accuracy of only a few centimetres. Centimetre accuracy is obtained based on the correction solution of L1 carrier phase ambiguities. However, these receivers are not suitable for use in dynamic systems such as unmanned

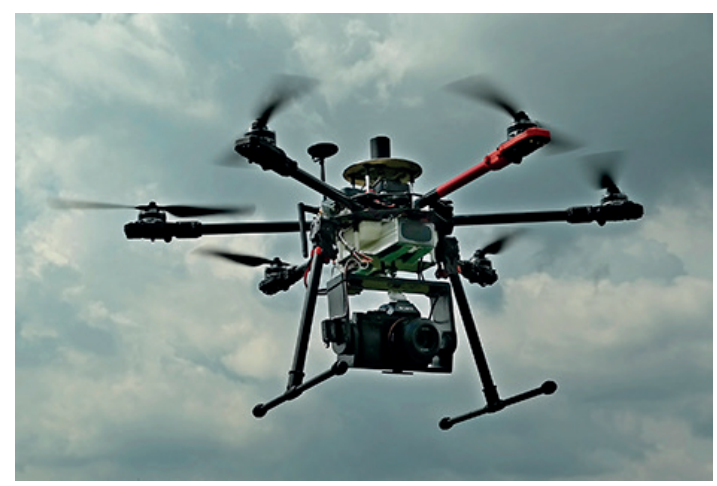

Fig. 1 Multirotor VTOL UAS BEE-G3 
aircraft. Poor reception may cause the loss of initialization and degradation of centimetre level positioning accuracy. Besides such L1 receivers, geodetic grade multi-frequency receivers are being developed with the continuous development of MST, as well as market requirements for smaller and more compact receivers. Currently, several dual frequencies GNSS receivers that are light-weight and specifically designed to be integrated into UAS can be found on the market. These receivers can obtain multi-frequency signals from GPS, GLONASS, BEIDOU and Galileo satellites. The price of such receivers is decreasing although it is still relatively high. In this case, the GNSS system serves a dual purpose. First, it serves as the NS (Navigation Sensor) for planning and performing of missions, and further as the PS (Position Sensor) for determining the position of the image sensor. In this example, the position is derived from a dual-frequency Septentrio AsteRx-m UAS GNSS receiver.

Corrected positioning solutions can be obtained as PPK (Post Processing Kinematic) or RTK (Real Time Kinematic) solutions. In the case of RTK, it is necessary to transmit RTCM (Radio Technical Commission for Maritime Services) corrections from a base station to the moving platform [25]. The base station can be a receiver located at a known point or a state GNSS reference correction network service distributed via NTRIP (Networked Transport of RTCM via Internet Protocol). In most applications, these differential corrections are passed to the rover's receiver in real-time requires a reliable communication link between the base and the rover. On the other hand, for UAS applications, this can cause a problem because the instrumentation required for an additional communication link to a base station can add significantly to the payload and power consumption. In order to achieve accuracy of a few centimetres baseline length must not be more than $5 \mathrm{~km}$ [1]. Post-processing of the UAS rover data with the base station data after the mission eliminates the need for a real-time data link between the UAS and base station, which simplifies the on-board setup and also reduces the payload and flight time. This additionally removes a potential source of interference in the connection that can occur during RTK solution. Reducing external effects increases system reliability.

The system used in this research has the ability to determine the 3D trajectory of the platform with PPK. The raw data is stored on a memory card that is embedded on-board the receiver of the moving platform. Data from the base station can be simultaneously stored in raw data format or downloaded afterwards from a network service. Then it is possible to post-process along with rover data to obtain the 3D trajectory of flight and coordinates of events with spatial accuracy. These coordinates represent the initial position of captured images.

\subsection{Camera calibration}

The UAS used in this research is equipped with a Sony Alpha 7R digital camera with a 36.3-megapixel full-frame $(35.9 \mathrm{~mm} \times 24 \mathrm{~mm})$ CMOS sensor and a Sony FE $35 \mathrm{~mm}$ high-quality Carl Zeiss lens (Table 1). The camera was calibrated on the test field that consists of 105 evenly distributed points. Test field coordinates were determined by the spatial intersections taken from several occupation-points measured by the TS Trimble S8. The achieved accuracy of the test field coordinates is $\pm 0.1 \mathrm{~mm}$ [26]. For determining interior orientation parameters, the phototriangulation with the self-calibration process was used. Phototriangulation was calculated with BBA method (Bundle Block Adjustment) by using Pix4D software. Input parameters in the adjustment are manually measured image coordinates of test field points, test field ground coordinates and initial interior parameters calculated from the camera and lens manufacture data. The self-calibration process implies conducting phototriangulation where elements of interior orientation parameters (focal length - f; the principal point position $-\mathrm{x}, \mathrm{y}$; and distortion elements of the camera lens) are introduced as unknowns into the adjustment. Furthermore, the test field coordinates, as well as image exterior orientation parameters (EOP) are unknown in the adjustment. Brown's lens model [27] with tree parameters for radial $\left(\mathrm{R}_{1}, \mathrm{R}_{2}, \mathrm{R}_{3}\right)$ and two for tangential $\left(\mathrm{T}_{1}, \mathrm{~T}_{2}\right)$ distortion was used. Numerous authors have researched the self-calibration process [28-31]. Camera interior orientation parameters obtained by phototriangulation with self-calibration based on the BBA method are shown in Table 2.

Table 1 Components of UAS

\begin{tabular}{lc}
\hline Frame & Tarrot \\
Battery & Lithium Polymer \\
Propulsion system & T-motors \\
Flight controller & Pixhawk v1 \\
GNSS receiver & Septentrio AsteRx-m UAS \\
Digital camera & Sony Alpha 7 \\
Lens & Sony FE 35mm \\
\hline
\end{tabular}


Table 2 Interior orientation parameters of Sony Alpha 7R with Sony FE $35 \mathrm{~mm}$ Carl Zeiss lens

\begin{tabular}{lcc}
\hline Parameter & Value $(\mathrm{px})$ & $\mathrm{SD}(\mathrm{px})$ \\
\hline Focal length $-\mathrm{f}$ & 7443.89 & 20.026 \\
Principal Point $-\mathrm{x}$ & 3659.19 & 14.930 \\
Principal Point $-\mathrm{y}$ & 2429.18 & 18.620 \\
Radial distortion $-\mathrm{R}_{1}$ & 0.050 & 0.00096 \\
Radial distortion $-\mathrm{R}_{2}$ & -0.215 & 0.00259 \\
Radial distortion $-\mathrm{R}_{3}$ & -0.024 & 0.00509 \\
Tangential distortion $-\mathrm{T}_{1}$ & -0.001 & 0.00049 \\
Tangential distortion $-\mathrm{T}_{2}$ & 0.001 & 0.00049 \\
\hline
\end{tabular}

\subsection{Camera trigger synchronization}

The camera is mounted on a custom-made servo-powered 2-axis gimbal with vibration dampers. In addition to determining position, the Septentrio AsteRx-m UAS GNSS system allows time registration of the camera shutter. With this information, it is possible to assign spatial coordinates to the shutter event. The procedure allows direct determination of camera position parameters at the centimetre level accuracy. The receiver time-stamps shutter events from the camera to precisely identify the times when the photographs were taken. These event markers along with the GNSS measurements are logged during the flight onto the on-board SD card for post-processing purposes. After the flight, data from the UAS and a base station reference receiver on the ground are post-processed. The derived centimetre level PPK position values are then embedded in the images, either directly in the EXIF data of the images, or in a separate CSV file. Coordinates of images contain camera trigger timing synchronization error.

\subsection{Lever arm correction}

Special attention was given to the design and installation of the sensors on the aircraft to minimize the impact of systematic errors in positioning between the GNSS antenna phase centre and the camera sensor. Necessary calibration of the system was performed in order to determine the initial position and elevation offsets between the camera sensor and the phase centre of the GNSS antenna [10]. After the calibration of the system, position offset is reduced at the level of several millimetres while height offset is set to 20.5 centimetres. These values are related when the platform is in a static position. With a dynamic platform, the positional offset between the sensors will degrade by increasing airspeed. Thus, at a speed of $3 \mathrm{~m} / \mathrm{s}$, the platform tilt in the motion direction is about 6 degrees corresponding to the difference in the initial position offset of $2 \mathrm{~cm}$ for pitch and roll. The impact of this error can be reduced by using "stop and go" method during the planning of the mission, but this reduces the autonomy and efficiency of the system. The error can also be reduced by the different design of the system and minimizing the vertical offset between the camera sensor and phase centre of the GNSS antenna. This approach in this research was to use tactical grade IMU data from the flight controller and compute lever arm correction due to the dynamic movement of the platform. Roll and pitch values are used for determining positional offset $\Delta$, while yaw or heading is used for calculation direction of offset Depending on the direction, corrections are added or subtracted from the initial positional coordinates. First of all, it is necessary to transform coordinates from WGS84 to the Cartesian coordinate system. The approximate attitude values of the platforms can be determined based on flight data log file and GNSS time for each individual shutter event. Based on the known distance between the camera and the antenna, roll and pitch angles it is possible to determine the approximate values for $\Delta d_{\varphi}$ and $\Delta d_{\psi}$ (Fig. 2(a), 2(b)) corrections due to inclination of moving platform. Furthermore, the correction values are calculated with respect to the flight direction frame. In order to add or subtract $\Delta E$ and $\Delta N$ corrections from initial coordinate values they must be re calculated in the Cartesian coordinate system (Fig. 2(c)).

\subsection{Spatial resolution investigation}

In addition to the $\mathrm{CP}$ (Check Points) comparison, spatial resolution was investigated, as this represents the quality of a UAS photogrammetry derived product that represents the interpretative capabilities of the sensor. The spatial resolution of images depends on several factors: flight altitude, focal length, optical quality, sensor resolution, atmospheric conditions and aircraft vibration. Spatial resolution can be defined with two values - Ground Sample Distance (GSD) and Ground Resolve Distance (GRD). GSD refers to the distance between pixels on the derived end product and represents a theoretical value. The actual resolution refers to the size of the smallest element distinguishable on the acquired imagery and depends on the factors that limit the system [32]. GRD is defined by line-pairs per mm that can be distinguished on an image of a test chart.

The RGB sensor of the Sony Alpha 7R camera was tested using a tri-bar test pattern (Fig. 3). The main reason for using this approach to test the UAS sensor is the simple geometry that is easy to construct. The target consists of parallel black lines positioned against a white background. 


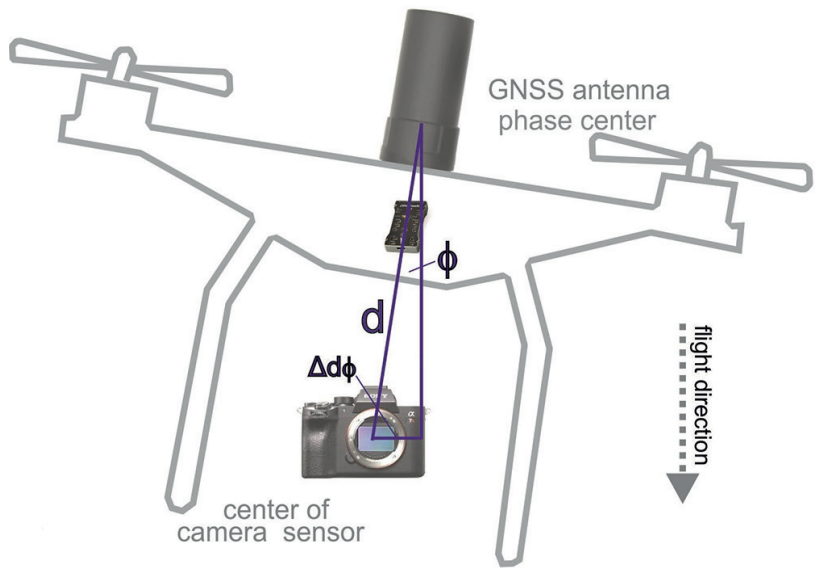

(a)

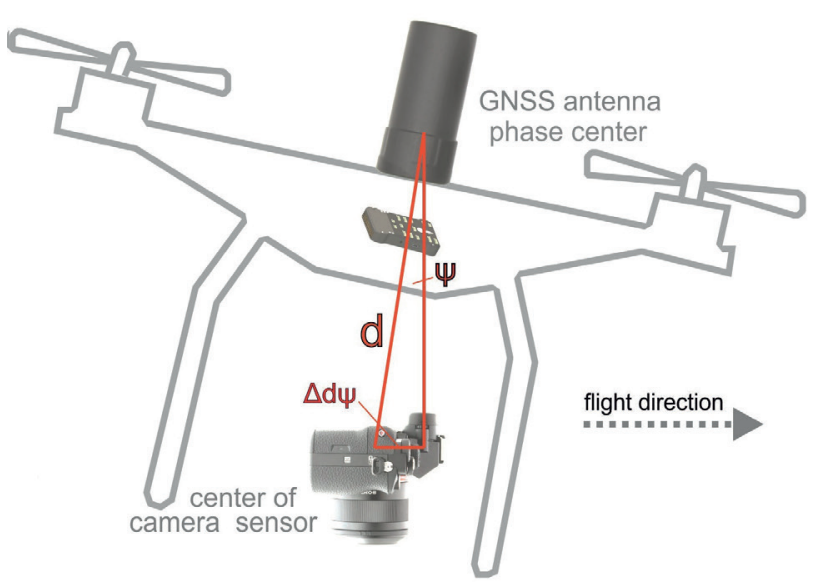

(b)

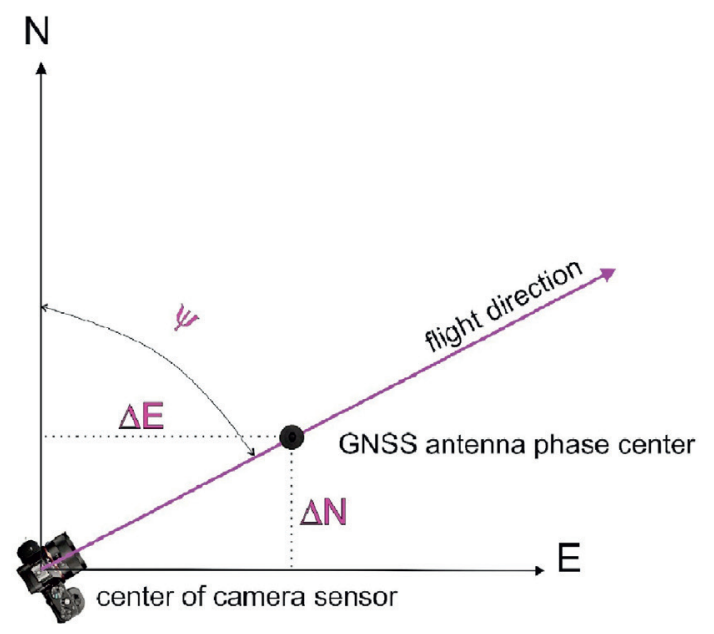

(c)

Fig. 2 a) correction $\Delta d_{\varphi}$ for roll angle perpendicular to flight direction, b) correction $\Delta d_{\psi}$ for pitch angle in flight direction and c) heading corrections $\Delta E, \Delta N$

The height of the lines is equal to spaces between them, while their width is seven times their height. This test pattern is reproduced at varied sizes to form an array consisting of bars of differing widths and spacing.

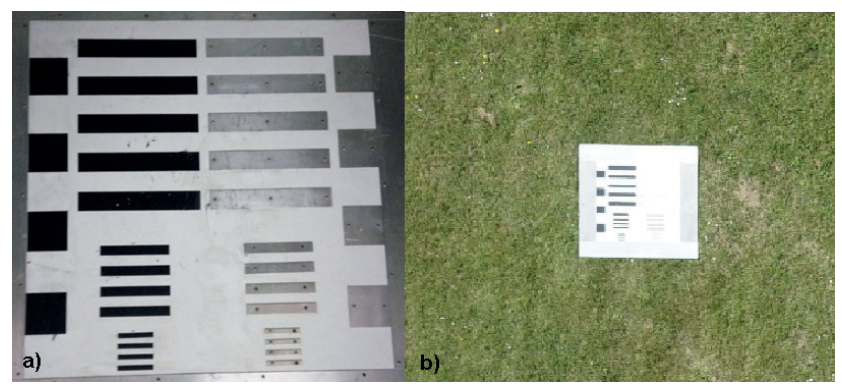

Fig. 3 a) Test pattern used for spatial resolution test, b) test pattern captured from UAS at 60 meters above the ground

GRD is an important sensor quality because it directly affects the planning of optimal mission parameters in accordance with the requirements of a project task. A line pairs per millimetre (LPM) is a parameter used to assess the spatial resolution of a sensor. Essentially, it is a mean of quantifying, under controlled conditions, the estimate of GRD by using a test chart pattern. The test chart pattern was placed in the middle of the test field in order to minimize the influence of lens distortion. Repetition of the pattern at different scales assures that the image of the pattern will include at least one pattern, which is so small that individual lines and their spaces will not be fully resolved. Visual inspection requires the observer to identify the smallest group of bars that can be completely distinguished. However, the subjective nature of this assessment should be considered when using this approach. GRD can be translated into a measure of resolution by the following relationship:

$$
G R D=\frac{h}{f^{*} R}
$$

where $h$ is flying altitude above the terrain, $f$ is a focal length and $R$ is a system resolution in LPM units. For the system used in this study, GRD is 9 millimetres when the flying altitude is 60 meters above ground, while for an altitude of $120 \mathrm{~m}, \mathrm{GRD}$ is 17 millimetres. From this, it can be concluded that GRD $=2 \times$ GSD. Based on the obtained values it can be concluded that the GSD should be half the value of the smallest object that needs to be resolved.

\subsection{Test field Slavonski Brod}

Test flights were performed on a flight area located near the city of Slavonski Brod. The selected test field is about 3 hectares. In order to test the UAS, 17 CPs were set at distances of 20 to $30 \mathrm{~m}$. CPs were made of plastic with dimensions $20 \times 20 \mathrm{~cm}$. Coordinates of CPs were determined based on observations with TS and prism. The Trimble S8 TS, 
which was used in the study has a precision of angle reading 1 second and measuring the distance of $1 \mathrm{~mm}+1 \mathrm{ppm}$. The TS was set and oriented with respect to fixed points in the Croatian terrestrial reference system HTRS96/TM with reference elevation system HVRS71. The targets were measured using the same reference frame.

$\mathrm{CP}$ were used only for further coordinate analysis in relation to the photogrammetric approach. The GNSS base station was set to the reference point from which we performed the terrestrial measurements with the TS. The distance of this reference point is about 100 meters from the closest $\mathrm{CP}$ at the test field. Placing the base station at the reference point of terrestrial measurements and using the unique coordinate system reduces the influence of random and systematic errors of $\mathrm{CP}$ estimation. Besides the installation of $\mathrm{CPs}$, a resolution test chart was placed near the centre of the test in order to analyze and estimate the true resolution, which defines the mapping capabilities of the system.

A series of flights with the different setup was carried out in the test field. The flights were performed within a single day with good lighting conditions and mild wind speeds. Each flight covered the test field with strips with $80 \%$ endlap and $80 \%$ sidelap between images.

First setup was with a flight altitude of $40 \mathrm{~m}$ above the ground, which corresponds to a GSD of $5 \mathrm{~mm}$. During the missions airspeed was around $3 \mathrm{~m} / \mathrm{s}$. Second flight is performed with similar flying directions to the first but at an altitude of $80 \mathrm{~m}$, with an airspeed of $6 \mathrm{~m} / \mathrm{s}$ and corresponding GSD of $1 \mathrm{~cm}$ (Fig. 4). Two data sets were derived based on these flights. Each data set consists of four flights with an identical setup. For each data set, estimated CP coordinates were compared with values obtained by terrestrial measurement.

\subsection{Test field Krk}

First test measurements were performed in almost ideal field conditions, which do not represent real field conditions that can be expected in everyday work. At the first test field, the topography of terrain is flat, there are no external disturbances that could interfere with the reception of GNSS signal while the influence of wind speed is minimal. Unlike the first test area with a slight change in the elevation of the terrain configuration, the area of the Test field Krk is rougher. Test field (Fig. 5) is about 11 ha with the highest elevation difference of around 20 meters. In order to maintain a unique scale, it is necessary to adjust the mission plan with respect to the elevation difference.

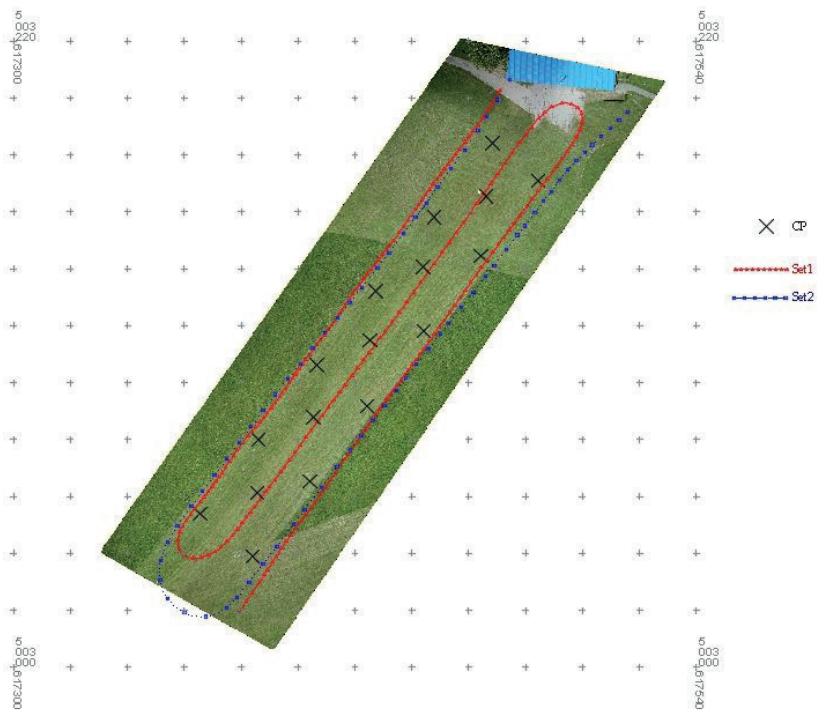

Fig. 4 The trajectory of flights at the test field Slavonski Brod
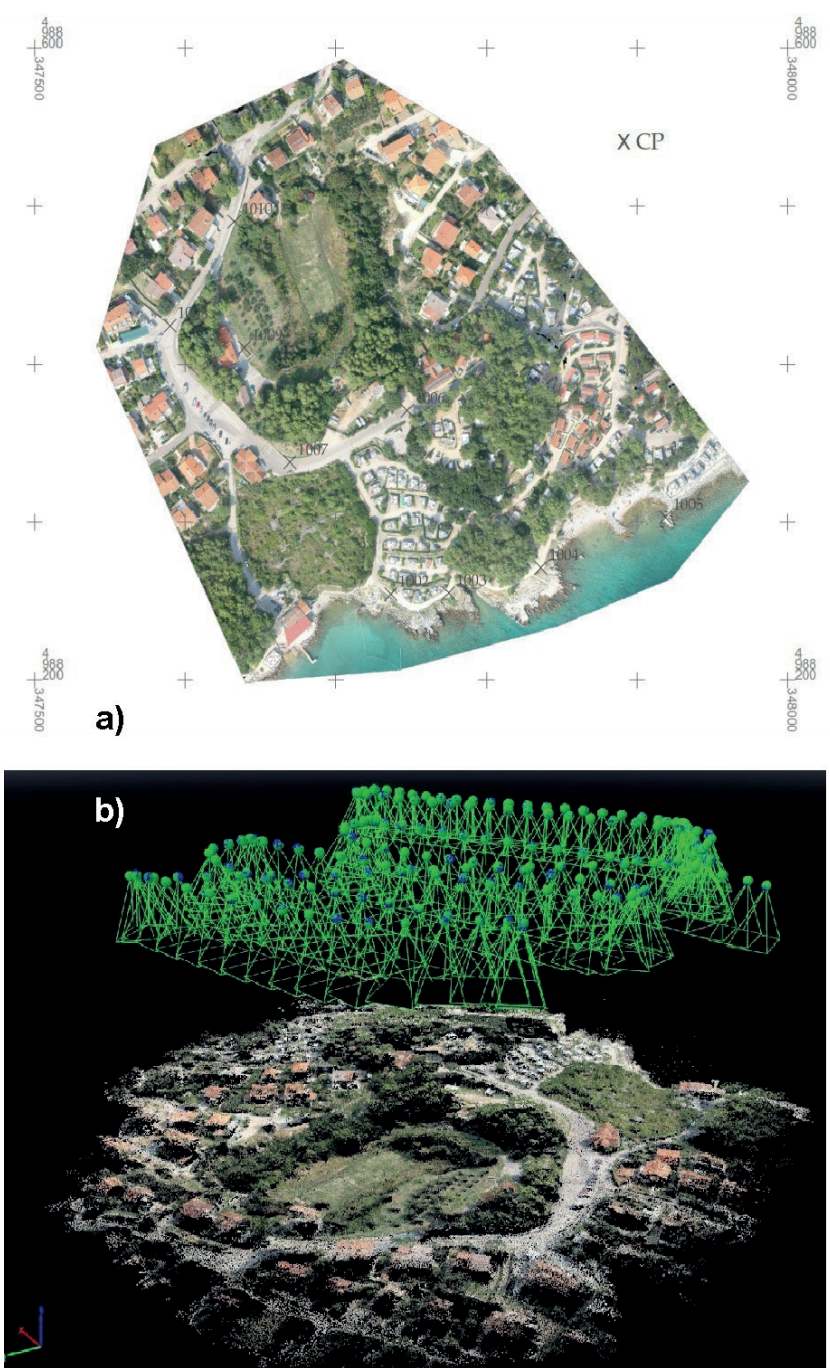

Fig. 5 a) Orthomosaic with GNSS measured feature control points, b) point cloud with camera locations of the test field Krk 
Above Ground Level (AGL) of the mission is set to $150 \mathrm{~m}$ with correspondent GSD of $20 \mathrm{~mm}$. The area was covered with seven strips with $80 \%$ endlap and $75 \%$ sidelap between images. The test area is located in the coastal area so that the surface of the sea influences the multipath of the GNSS signal while the wind speed is variable with ranges from $2 \mathrm{~m} / \mathrm{s}$ to $6 \mathrm{~m} / \mathrm{s}$. Multipath and variable wind speed effects on GNSS position accuracy.

In this research, point coordinates of characteristic features at the test field will be compared concerning terrestrial measurement. These nine features were manually selected based on identification possibilities with high-resolution orthomosaic (Fig. 6). Feature CPs were measured with the Trimble R10 GNSS system using Croatian Positioning Virtual Reference System (CROPOS VRS) online service [5] with position and elevation accuracy of $2 \mathrm{~cm}$ and $4 \mathrm{~cm}$ (Fig. 6).

\subsection{Data processing and accuracy assessment}

Raw data observations from the base and moving (UAS) GNSS receivers were processed to obtain spatial information of each image shutter event. The positional accuracy of images is between 2 and 3 centimetres while vertical accuracy is from 3 to 4 centimetres. Geo-tagged images with centimetre level accuracy are used as the input parameter for scene reconstruction of the two above mentioned test fields. Photogrammetric processing of test field was performed with photogrammetric software: PiX4D (version: 4.0.18) designed for processing of aerial images from UAS. Image processing is comprised of several steps. Crucial steps during image processing are Scale Invariant Feature Transform (SIFT) feature matching and bundle block adjustment. Automatic Aerial Triangulation (AAT) is based on the SIFT algorithm which extracts matching points for individual images. These matching points along with the camera position are used in bundle block adjustment to reconstruct the exterior orientation parameters (position and orientation) for each individual image. Both software have optimized SIFT algorithms and scene reconstruction processes to achieve better results. Finally, the generated photorealistic 3D model was used for measuring the CP coordinates.

After processing the data from test field Slavonski Brod, the coordinates of CP were estimated for each individual flight data set. Same, above mentioned data processing workflow, was used for Test field Krk data. The coordinates of the manually selected characteristic features $(\mathrm{CP})$ on the ground were estimated based on the photorealistic 3D model. Obtained coordinates were compared with the CP coordinates collected by the terrestrial geodesy measurement with a total station and GNSS RTK. For accuracy assessment, the mean error $(M E)$ of differences between terrestrial and aerial coordinates for data set was used. Standard deviation (SD) shows the variation of $M E$. Root mean square error (RMSE) in geodesy indicate accuracy of the system while maximum (Max) and minimum (Min) provide a limit of coordinate comparison values. Statistical parameters were computed separately for each axis $(E, N, H)$ based the following equations given for $E$ direction:

$$
\begin{aligned}
& \Delta E_{i}=E_{\text {GNSS }, i}-E_{\text {aerial }, i}, \\
& M E_{E}=\frac{\sum_{i=1}^{n} \Delta E_{i}}{n}, \\
& S D_{E}=\sqrt{\frac{\sum_{i=1}^{n}\left(\Delta E_{i}-M E_{E}\right)^{2}}{n-1},} \\
& \operatorname{RMSE}_{E}=\sqrt{\frac{\sum_{i=1}^{n}\left(\Delta E_{i}\right)^{2}}{n},}
\end{aligned}
$$

where $\Delta$ is the difference between $\mathrm{CP}$ measured with GNSS receiver or total station on the field and coordinates derived from UAS imagery and $\mathrm{n}$ is a total number of CP. Entire data processing results, as well as accuracy assessments and comparison of the results for both test fields, is shown in the next section.

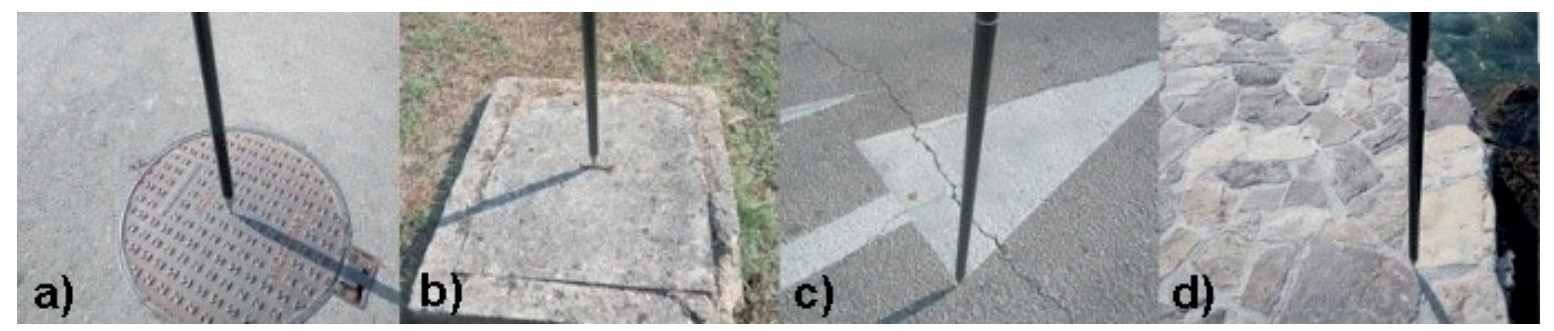

Fig. 6 Terrestrial GNSS measurements of the feature CP: a) circular manhole cover, b) squared manhole cover, c) road surface marker, d) marine query 


\section{Results}

As previously mentioned, the main goal of this research analyses the spatial data positioning and resolution performance test of UAS with direct georeferencing and application of SfM photogrammetry. In Table 3 statistical parameters of the BBA for all phototriangulation procedures in this research are shown.

The Table 4 shows that the values of differences between CP coordinates before and after lever arm correction based on IMU data from the flight controller. Root mean square error (RMSE) represents a measure for spatial accuracy of field geodesy that includes systematic and random errors. As we can see in Fig. 7 error in the vertical direction is greater for the second set. The reason for the

Table 3 Statistical parameters of the BBA for all adjustments on Slavonski brod and Krk test fields

\begin{tabular}{|c|c|c|c|}
\hline \multirow{2}{*}{ Test field } & \multicolumn{2}{|c|}{ Slavonski brod } & \multirow{2}{*}{ Krk } \\
\hline & Set1 & Set2 & \\
\hline No. of acquired images & 82 & 33 & 174 \\
\hline No. of adjusted images & 69 & 30 & 172 \\
\hline $\begin{array}{l}\text { No. of images with fixed } \\
\text { ambiguate }\end{array}$ & 82 & 33 & 174 \\
\hline No. of GNSS satellites & 12 & 12 & 11 \\
\hline Number of CP & 17 & 17 & 8 \\
\hline GSD (cm) & 0.52 & 1.05 & 1.97 \\
\hline Processed coverage area (ha) & 3 & 3 & 13 \\
\hline No. of strips & 3 & 2 & 7 \\
\hline Flight time (minutes) & 5 & 6 & 24 \\
\hline Endlap/Sidelap & $80 / 80$ & $80 / 80$ & $80 / 75$ \\
\hline Mission planned AGL & 40 & 80 & 150 \\
\hline Reprojection error (pixels) & 0.156 & 0.171 & 0.237 \\
\hline $3 \mathrm{D}$ tie points for BBA & 363433 & 245554 & 1896592 \\
\hline Processing time & $1: 15: 21$ & $0: 37: 35$ & $4: 38: 43$ \\
\hline
\end{tabular}

Table 4 Comparison of results of lever arm correction between data sets from the test field Slavonski Brod. Values are presented in millimetres

\begin{tabular}{|c|c|c|c|c|c|c|c|}
\hline & & \multicolumn{3}{|c|}{ Set1 } & \multicolumn{3}{|c|}{ Set2 } \\
\hline & & E & $\mathrm{N}$ & $\mathrm{H}$ & E & $\mathrm{N}$ & $\mathrm{H}$ \\
\hline \multirow{5}{*}{$\stackrel{\text { : }}{\stackrel{\Xi}{\Xi}}$} & $\mathrm{ME}$ & -14 & -34 & 41 & 37 & -11 & 64 \\
\hline & SD & 25 & 28 & 34 & 17 & 23 & 49 \\
\hline & RMSE & 28 & 43 & 52 & 41 & 24 & 79 \\
\hline & Max & 12 & 7 & 81 & 59 & 19 & 147 \\
\hline & Min & -67 & -82 & -17 & -2 & -45 & 5 \\
\hline \multirow{5}{*}{$\begin{array}{l}\overrightarrow{0} \\
\stackrel{0}{0} \\
\stackrel{0}{0} \\
ن\end{array}$} & $\mathrm{ME}$ & -8 & -16 & 38 & 17 & -7 & 48 \\
\hline & SD & 14 & 15 & 31 & 11 & 13 & 24 \\
\hline & RMSE & 16 & 19 & 42 & 19 & 14 & 54 \\
\hline & Max & 8 & 5 & 78 & 32 & 10 & 94 \\
\hline & Min & -26 & -29 & -15 & 4 & -21 & 4 \\
\hline
\end{tabular}

greater error is that the offset between the GNSS antenna phase centre and camera sensor increases due to higher airspeed. Vertical error is due to its complexity not considered as a subject of this research.

The data processing procedure used for the second test field on the Island of Krk was equivalent to the first test field described above. The position accuracy of the images was 2 to $4 \mathrm{~cm}$, while the elevation accuracy was 3 to $5 \mathrm{~cm}$. Terrestrial GNSS measurements of feature CPs were not used for 3D reconstruction but for the comparison with respect to the estimated coordinates of the feature. The differences of point coordinates are presented in millimetres and shown in Table 5.

Based on the values in Table 4 and Fig. 7, it can be concluded that spatial data can be obtained with the accuracy of several centimetres. Coordinate differences between features CPs are larger with respect to the plastic CPs used

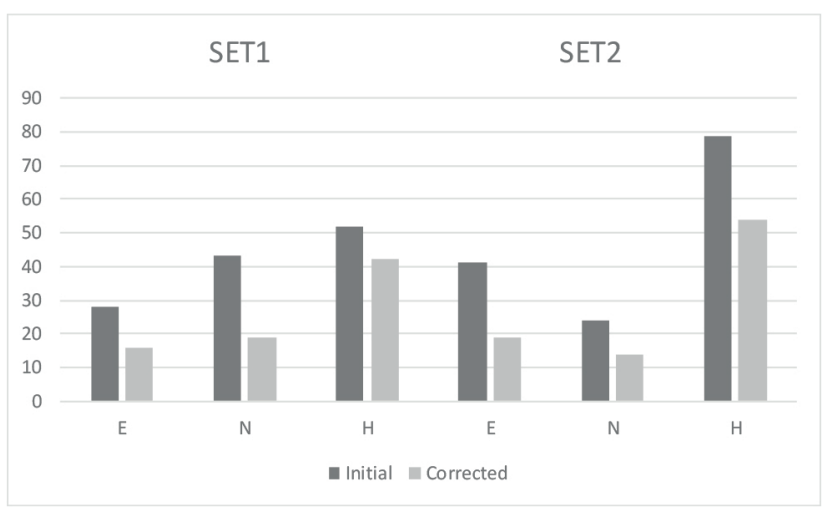

Fig. 7 Distribution of RMSE [mm] before and after lever arm correction

Table 5 Results of the coordinate comparison at the test field Krk. Values are presented in millimetres

\begin{tabular}{lcccc}
\hline Features & $\mathrm{E}$ & $\mathrm{N}$ & $\mathrm{H}$ & $3 \mathrm{D}$ \\
\hline circular manhole cover & 20 & -7 & -6 & 22 \\
squared manhole cover & -12 & -21 & -19 & 31 \\
road surface marker & 19 & -10 & 64 & 68 \\
marine query & -11 & 22 & -47 & 53 \\
squared manhole edge & 12 & -15 & -16 & 25 \\
red cross marker & 13 & 13 & 41 & 44 \\
asphalt edge & -9 & -8 & -19 & 22 \\
plateau edge & 11 & 13 & -20 & 26 \\
road edge & -24 & 11 & 58 & 63 \\
ME & 2 & 0 & 4 & 39 \\
SD & 15 & 14 & 37 & 17 \\
RMSE & 23 & 21 & 56 & 64 \\
Min & -24 & -21 & -47 & 22 \\
Max & 20 & 22 & 64 & 68 \\
\hline
\end{tabular}


at the test field Slavonski Brod. Planar accuracy is between 2 and 3 centimetres while spatial accuracy is up to 7 centimetres. The main reason for this is the degradation of elevation accuracy.

\section{Discussion and conclusions}

This study aims to examine the possibility of using midcost Unmanned Aircraft System (UAS) with geodetic grade multi-frequency receiver for precise camera positioning and modern photogrammetric software for 3D scene reconstruction, which satisfied contemporary spatial data requirements in geodesy. Test measurements confirmed the accuracy at the level of few centimetres which can be achieved using the described UAS in both tests. Thus, tested custom made UAS is applicable for various surveying purposes, such as cadastral, but also for topographic and engineering purposes, as well as for the infrastructure inspection and monitoring purposes. The described method is particularly useful in areas inaccessible for classical terrestrial geodetic methods. The spatial

\section{References}

[1] Colomina, I., Molina, P. "Unmanned aerial systems for photogrammetry and remote sensing: A review", ISPRS Journal of Photogrammetry and Remote Sensing, 92, pp. 79-97, 2014. https://doi.org/10.1016/j.isprsjprs.2014.02.013

[2] Kordić, B., Lužar-Oberiter, B., Pikelj, K., Matoš, B., Vlastelica, G. "Integration of Terrestrial Laser Scanning and UAS Photogrammetry in Geological Studies: Examples from Croatia", Periodica Polytechnica Civil Engineering, 63(4), pp. 989-1003, 2019. https://doi.org/10.3311/PPci.14499

[3] Küng, O., Strecha, C., Beyeler, A., Zufferey, J.-C., Floreano, D., Fua, P., Gervaix, F. "The accuracy of automatic photogrammetric techniques on ultra-light UAV imagery", International Archives of the Photogrammetry, Remote Sensing and Spatial Information Sciences, XXXVIII-1/C22, pp. 125-130, 2011. https://doi.org/10.5194/isprsarchives-XXXVIII-1-C22-125-2011

[4] Reshetyuk, Y., Mårtensson, S.-G. "Generation of Highly Accurate Digital Elevation Models with Unmanned Aerial Vehicles", The Photogrammetric Record, 31(154), pp. 143-165, 2016. https://doi.org/10.1111/phor.12143

[5] Vallet, J., Panissod, F., Strecha, C., Tracol, M. "Photogrammetric Performance of an Ultra Light Weight Swinglet "UAV", International Archives of the Photogrammetry, Remote Sensing and Spatial Information Sciences, XXXVIII-1/C22, pp. 253-258, 2011. https://doi.org/10.5194/isprsarchives-XXXVIII-1-C22-253-2011

[6] Pikelj, K., Ružić, I., Ilić, S., James, M. R., Kordić, B. "Implementing an efficient beach erosion monitoring system for coastal management in Croatia", Ocean \& Coastal Management, 156, pp. 223$238,2018$.

https://doi.org/10.1016/j.ocecoaman.2017.11.019 resolution test proves that the real resolution of the system is dependent on the Ground Resolve Distance (GRD), which is twice the Ground Sample Distance (GSD). In this approach, images were captured in aerial or nadir view. Future work should be focused on testing the ability of the system with images captured in oblique or free flight capture mode. The accuracy of the camera position is reduced due to a timing error. Therefore, future research should be focused on reducing the impact of time synchronization. Furthermore, the relative distance between the camera sensor and phase centre of the antenna is a fixed value in this research. However, this is not the case of a dynamic platform as the gimbal corrects the camera to a zenith view. The influence of the gimbal, as well as the vertical correction, could be investigated by additional inertial measure unit (IMU) mounted on the gimbal.

\section{Acknowledgement}

We would like to thank pilot Milivoj Hucaljuk for UAS calibration and mission planning support.

[7] Navarro, J., Parés, M. E., Colomina, I., Bianchi, G., Pluchino, S., Baddour, R., Consoli, A., Ayadi, J., Gameiro, A., Sekkas, O., Tsetsos, V., Gatsos, T., Navoni, R. "A Redundant GNSS-INS LowCost UAV Navigation Solution for Professional Applications", International Archives of the Photogrammetry, Remote Sensing and Spatial Information Sciences, XL-3/W3, pp. 299-306, 2015. https://doi.org/10.5194/isprsarchives-XL-3-W3-299-2015

[8] Martínez, M., Blázquez, M., Gómez, A., Colomina, I. "A new approach to the use of position and attitude control in camera orientation", presented at 7th International Geomatic Week, Barcelona, Spain, Feb. 20-23, 2007. [online] Available at: https://www. researchgate.net/publication/268286865_a_new_approach_to_the use_of_position_and_attitude_control_in_camera_orientation

[9] Rosnell, T., Honkavaara, E. "Point Cloud Generation from Aerial Image Data Acquired by a Quadrocopter Type Micro Unmanned Aerial Vehicle and a Digital Still Camera", Sensors, 12(1), pp. 453480, 2012.

https://doi.org/10.3390/s120100453

[10] Pfeifer, N., Glira, P., Briese C. "Direct georeferencing with on board navigation components of light weight UAV platforms", International Archives of the Photogrammetry, Remote Sensing and Spatial Information Sciences, XXXIX-B7, pp. 487-492, 2012. https://doi.org/10.5194/isprsarchives-XXXIX-B7-487-2012

[11] Rehak, M., Mabillard, R., Skaloud, J. "A micro-UAV with the capability of direct georeferencing", International Archives of the Photogrammetry, Remote Sensing and Spatial Information Sciences, XL-1/W2, pp. 317-323, 2013. https://doi.org/10.5194/isprsarchives-XL-1-W2-317-2013 
[12] Mian, O., Lutes, J., Lipa, G., Hutton, J. J., Gavelle, E., Borghini, S. "Direct georeferencing on small unmanned aerial platforms for improved reliability and accuracy of mapping without the need for ground control points", International Archives of the Photogrammetry, Remote Sensing and Spatial Information Sciences, XL-1/W4, pp. 397-402, 2015.

https://doi.org/10.5194/isprsarchives-XL-1-W4-397-2015

[13] Rehak, M. "Integrated Sensor Orientation on Micro Aerial Vehicles", Doctoral Thesis, Swiss Federal Institute of Technology in Lausanne, Switzerland, 2017. https://doi.org/10.5075/epfl-thesis-7530

[14] Stöcker, C., Nex, F., Koeva, M., Gerke, M. "Quality assessment of combined IMU/GNSS data for direct georeferencing in the context of UAV-based mapping", International Archives of the Photogrammetry, Remote Sensing and Spatial Information Sciences, XLII-2/W6, pp. 355-361, 2017.

https://doi.org/10.5194/isprs-archives-XLII-2-W6-355-2017

[15] Küng, O., Strecha, C., Fua, P., Gurdan, D., Achtelik, M., Doth, K.-M., Stumpf, J. "Simplified building models extraction from ultralight UAV imagery", International Archives of the Photogrammetry, Remote Sensing and Spatial Information Sciences, XXXVIII-1/ C22, pp. 217-222, 2011.

https://doi.org/10.5194/isprsarchives-XXXVIII-1-C22-217-2011

[16] Gini, R., Pagliari, D., Passoni, D., Pinto, L., Sona, G., Dosso, P. "UAV Photogrammetry: block triangulation comparisons", International Archives of the Photogrammetry, Remote Sensing and Spatial Information Sciences, XL-1/W2, pp. 157-162, 2013. https://doi.org/10.5194/isprsarchives-XL-1-W2-157-2013

[17] Remondino, F., Spera, M. G., Nocerino, E., Menna, F., Nex, F. "State of the art in high density image matching", The Photogrammetric Record, 29(146), pp. 144-166, 2014. https://doi.org/10.1111/phor.12063

[18] Chiabrando, F., Donadio, E., Rinaudo, F. "SfM for Orthophoto to Generation: A Winning Approach for Cultural Heritage Knowledge", International Archives of the Photogrammetry, Remote Sensing and Spatial Information Sciences, XL-5/W7, pp. 91-98, 2015. https://doi.org/10.5194/isprsarchives-XL-5-W7-91-2015

[19] Murtiyoso, A., Grussenmeyer, P. "Documentation of heritage buildings using close-range UAV images: dense matching issues, comparison and case studies", The Photogrammetric Record, 32(159), pp. 206-229, 2017. https://doi.org/10.1111/phor.12197

[20] Gašparović, M., Jurjević, L. "Gimbal Influence on the Stability of Exterior Orientation Parameters of UAV Acquired Images", Sensors, 17(2), Article number: 401, 2017. https://doi.org/10.3390/s17020401

[21] Carbonneau, P. E., Dietrich, J. T. "Cost-effective non-metric photogrammetry from consumer-grade sUAS: implications for direct georeferencing of structure from motion photogrammetry", Earth Surface Processes and Landforms, 42(3), pp. 473-486, 2017. https://oi.org/10.1002/esp.4012
[22] Gonçalves, G. R., Pérez, J. A., Duarte, J. "Accuracy and effectiveness of low cost UASs and open source photogrammetric software for foredunes mapping", International Journal of Remote Sensing, 39(15-16), pp. 5059-5077, 2018. https://doi.org/10.1080/01431161.2018.1446568

[23] Pérez, J. A., Gonçalves, G. R., Rangel, J. M. G., Ortega, P. F. "Accuracy and effectiveness of orthophotos obtained from low cost UASs video imagery for traffic accident scenes documentation", Advances in Engineering Software, 132, pp. 47-54, 2019. https://doi.org/10.1016/j.advengsoft.2019.03.010

[24] Turner, D., Lucieer, A., Wallace, L. "Direct Georeferencing of Ultrahigh-Resolution UAV Imagery", IEEE Transactions on Geoscience and Remote Sensing, 52(5), pp. 2738-2745, 2014. https://doi.org/10.1109/TGRS.2013.2265295

[25] Gerke, M., Przybilla, H.-J. "Accuracy Analysis of Photogrammetric UAV Image Blocks: Influence of Onboard RTK-GNSS and Cross Flight Patterns", Photogrammetrie - Fernerkundung Geoinformation, 1, pp. 17-30, 2016. https://doi.org/10.1127/pfg/2016/0284

[26] Gašparović, M., Gajski, D. "The algorithm for the precise elimination of lens distortion influence on digital cameras", Geodetski list, 70(93), pp. 25-38, 2016. (in Croatian) [online] Available at: https:// hrcak.srce.hr/156881

[27] Brown, D. C. "Decentering Distortion of Lenses", Photogrammetric Engineering, 32(3), pp. 444-462, 1966. [online] Available at: https://pdfs.semanticscholar.org/2ef0/01c656378a1c5cf80488b 35684742220d3f9.pdf?_ga $=2.133332230 .921945013 .1588680194-$ 1062025215.1588680194

[28] Pollefeys, M. "Self-calibration and metric 3D reconstruction from uncalibrated image sequences", Doctoral Thesis, Catholic University of Leuven, Leuven Belgium, 1999. [online] Available at: http://people.inf.ethz.ch/pomarc/pubs/smallpdfs/PollefeysPhD.pdf

[29] Gonçalves, J. A., Henriques, R. "UAV photogrammetry for topographic monitoring of coastal areas", ISPRS Journal of Photogrammetry and Remote Sensing, 104, pp. 101-111, 2015. https://doi.org/10.1016/j.isprsjprs.2015.02.009

[30] Babapour, H., Mokhtarzade, M., Zoej, M. J. V. "Self-calibration of digital aerial camera using combined orthogonal models", ISPRS Journal of Photogrammetry and Remote Sensing, 117, pp. 29-39, 2016. https://doi.org/10.1016/j.isprsjprs.2016.03.015

[31] Gašparović, M., Gajski, D. "Two-step camera calibration method developed for micro UAV's", International Archives of the Photogrammetry, Remote Sensing and Spatial Information Sciences, XLI-B1, pp. 829-833, 2016. https://doi.org/10.5194/isprs-archives-XLI-B1-829-2016

[32] Orych, A. "Review of methods for determining the spatial resolution for UAV sensors", International Archives of the Photogrammetry, Remote Sensing and Spatial Information Sciences, XL-1/W4, pp. 391-395, 2015.

https://doi.org/10.5194/isprsarchives-XL-1-W4-391-2015 